\title{
The suitability of groundwater in Mosul city for various civilizational uses.
}

\author{
Abdulmoneim M.A.Kannah ${ }^{1 *}$ \\ ${ }^{1 * B}$ Biological department, college sciences, Mosul university, Mosul, IRAQ \\ E-mail: abmsbio38@uomosul.edu.iq
}

(Received May 06, 2021; Accepted August 23, 2021; Available online December 01, 2021)

DOI: 10.33899/edusj.2021.129867.1155, (C 2021, College of Education for Pure Science, University of Mosul.

This is an open access article under the CC BY 4.0 license (http://creativecommons.org/licenses/by/4.0/).

\begin{abstract}
:
In the current research, 23 wells were chosen from some residential neighborhoods located in the city of Mosul. To study some of the physical properties of well water represented by temperature, electrical conductivity, and total dissolved salts, as well as, the study of chemical analyzes are: (sulfates, chlorides, phosphates, nitrates, sodium, potassium, calcium, magnesium, dissolved oxygen and $\mathrm{pH}$ ).

The results of the study refer to the height of the electrical conductivity, which ranged between (7912456) $\mu \mathrm{mhos} / \mathrm{cm}$. The results showed that a lot of water is free of dissolved oxygen, as it recorded the highest value of $4.6 \mathrm{mg} / \mathrm{L}$. Whereas, the temperature of the studied water ranged between (2028.4) $\mathrm{C}^{\mathrm{o}}$, and it is considered warm water. In the current study, the calcium ion concentration was greater than the magnesium ion concentration in all well water, and the highest concentration reached (264 and 134) $\mathrm{mg} / \mathrm{L}$ at well 22 and 23, respectively. There was an increase in the concentration of chloride ion, which reached (204) mg/L and the lowest concentration (32) mg/L at wells 11 and 7, respectively.

When comparing the values of the electrical conductivity of the well water with the global determinants of drinking, it was determined that they are not suitable for drinking.

When applying the relationship between the value of the electrical conductivity and the ratio of sodium adsorption to well water, it was found that all water from wells Class (C3 - S1) except for wells (11 and 19) are classified as Class (C4 - S1)
\end{abstract}

Keywords: Groundwater., Mosul city., Iraq.

$$
\begin{aligned}
& \text { مدى ملائمة نوعية مياه مدينة الموصل الجوفية لمختلف الاستخدامات المدنية . }
\end{aligned}
$$

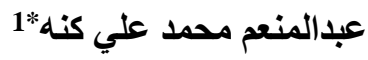

$$
\begin{aligned}
& \text { 11*قسم علوم الحياة، كلية العلوم، جامعة الموصل، الموصل، العراق } \\
& \text { الخلاصة : } \\
& \text { في البحث الحالي تم اختيار } 23 \text { بئرا من بعض الاحياء السكنية الواقعة ضمن مدينة الموصل لدراسة بعض الصفات الفيزيائية لمياه } \\
& \text { الابار والمتمثلة بدرجة الحرارة والتوصيلة الكهربائية والاملاح الكلية الذائبة , فضلا عن , دراسة التحاليل الكيميائية هي : ( الكبريتات }
\end{aligned}
$$

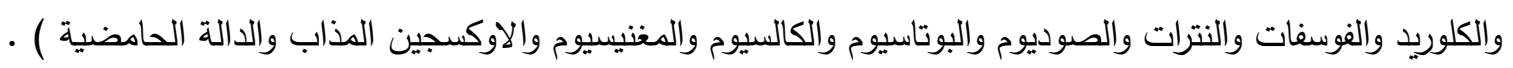


اشارت نتائج الدراسة الى ارتفاع التوصيلة الكهربائية والتي تراوحت بين (791 -2456) مايكروموز / سم وبينت النتائج خلو كثير

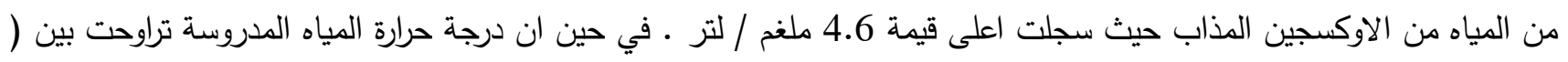

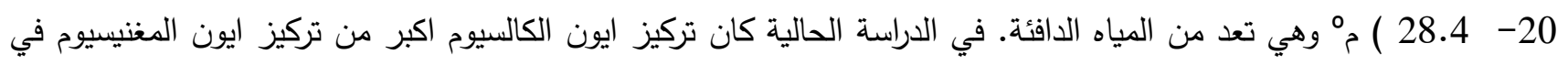

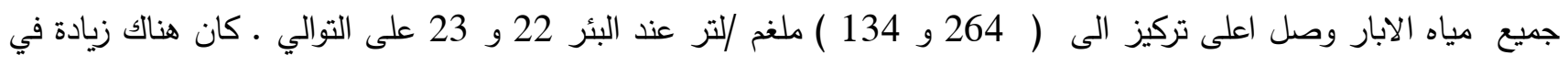
تركيز ايون الكلورايد حيث وصل الى ( 204 ) ملغم / لتر واقل تركيز (32 ) ملغم / لتر عند البئرين 11 و و7 على التئ التوالي.

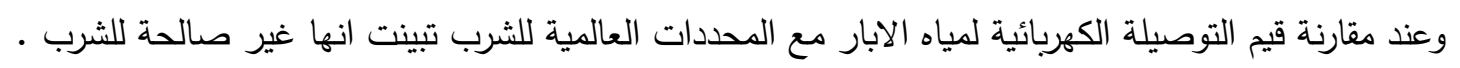

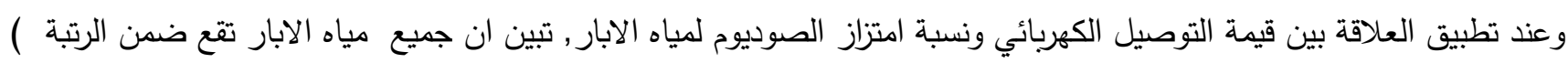

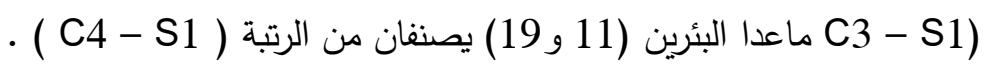

الكلمات الدالة: المياه الجوفية ., مدينة الموصل ., العراق •

المقدمة Introduction:

ان نسبة 71.7 \% من المياه الجوفية في دول الثرق الاوسط وشمال افريقيا هي صالحة للشرب, وتشمل مياه العيون والابار , ومصادر هذه المياه هي مياه الامطار والري والمياه السطحية والتي تتغلغل الى داخل التربة ووصولا الى الخزان المائي , ويبدأ الناس

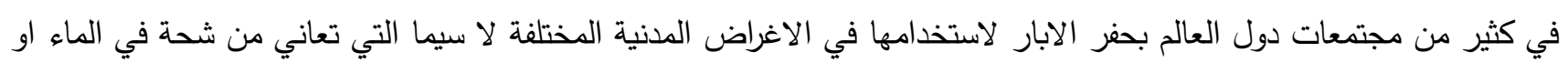

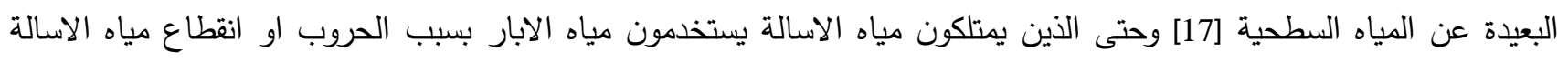

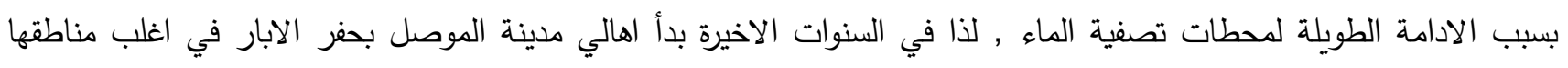

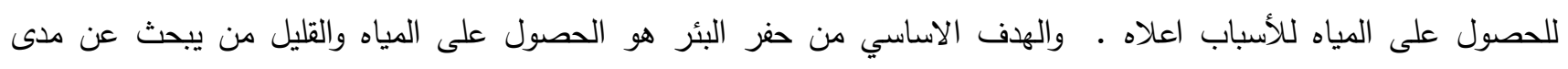
صلاحية هذه المياه للشرب او الزراعة او الصناعة. هناك دراسات كثيرة عن المياه الجوفية , وهي احدى الانظمة البيئية والمتصلة مع الانظمة البيئية الاخرى مما يحصل تغيير في

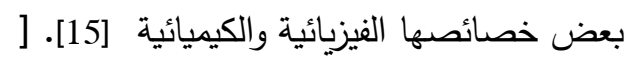
من الدراسات حول المياه الجوفية دراسة [6]. اذ بينت ان البئرين الواقعين في قريتي الخرار والخربة جنوب غرب التباتية الموصل واللتان

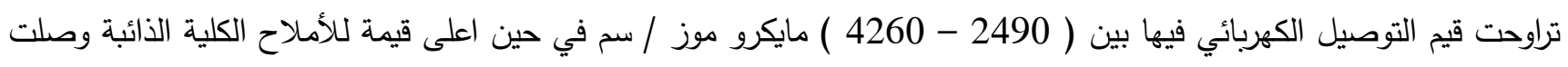

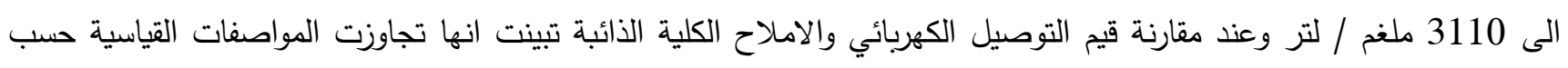

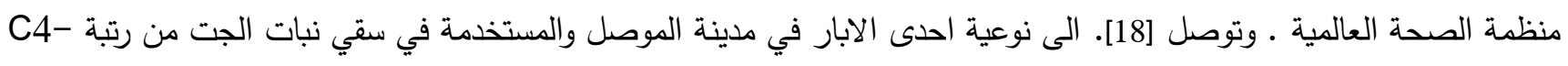
S1 عالي الملوحة جدا - قليل الصوديوم حسب تصنيف مختبر الملوحة الامريكي ويستخدم في ري المحاصيل ذات التحمل الملحي

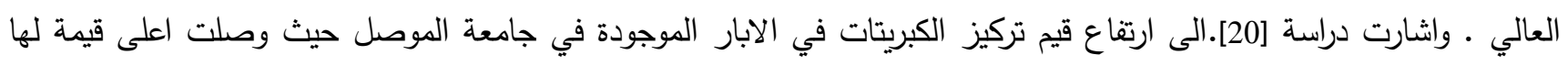

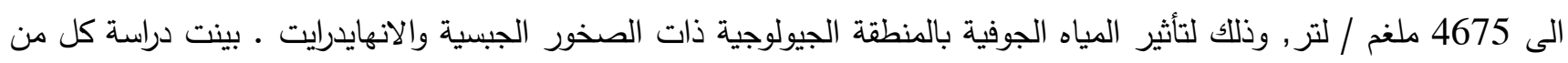

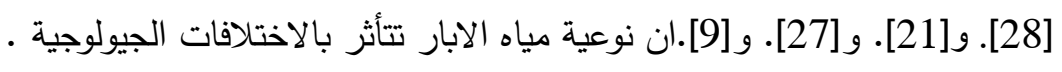
كان الهدف من الدراسة اختيار بعض الابار في مدينة الموصل للوقوف على نوعية هذه المياه ومدى ملائمتها للاستخدامات المدنية

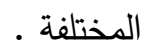

\section{المواد وطرائق العمل Material and Methods :} اختير 23 بئرا موزع بشكل عشوائي في مدينة الموصل الثكل (1) وجمعت العينات خلال فصل الخريف من عام 2019 كمسح ميداني لبعض الاحياء السكنية من مدينة الموصل , وبعد تثغيل مضخة سحب المياه لمدة بين (5-10) دقائق جمعت عينات مياه 
الابار والتي لم يتجاوز عمقها عن 35 مترا , وبعد وضع المياه في قناني بلاستيكية سعة واحد لتر , اجريت عليها التحاليل الفيزيائية

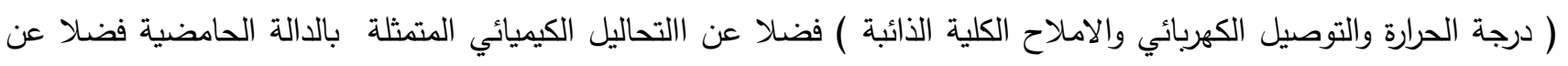
الايونات السالبة ( الاوكسجين المذاب والكبريتات والنترات والفوسفات والكلوريدات ) والايونات الموجبة ( الكالسيوم والمغنيسيوم والصوديوم والبوتاسيوم ), واجريت التحاليل الفيزيائية والكيميائية في مختبرات علوم الحياة - كلية العلوم - جامعة الموصل ,

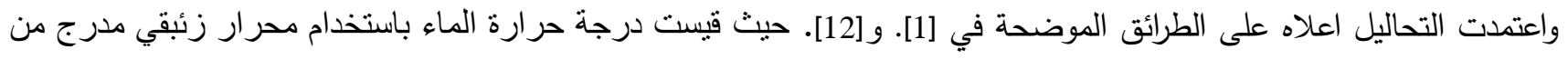

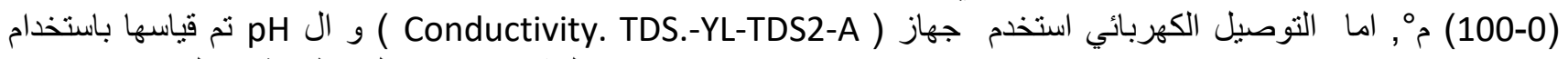

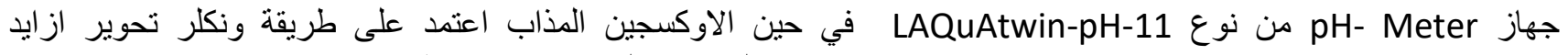

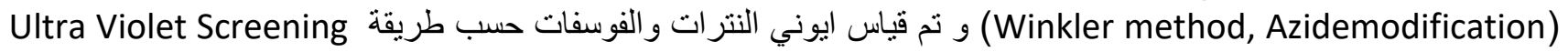

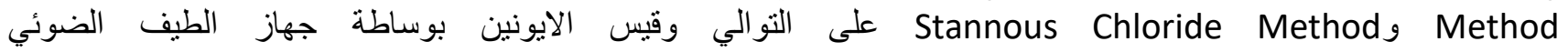
Spectrophotometer

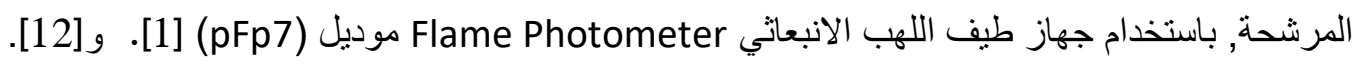

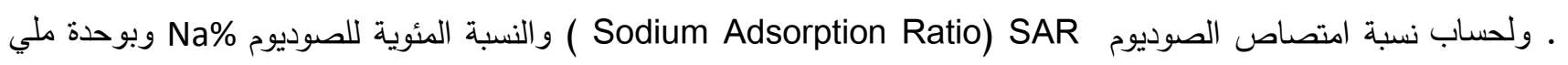

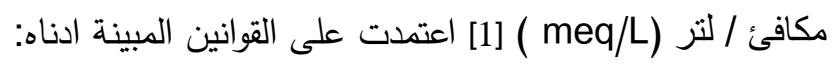

$\mathrm{Na}$

S.A.R $=\frac{}{\sqrt{\frac{\mathrm{Ca}+\mathrm{Mg}}{2}}} \quad \mathrm{Na} \%=\frac{\mathrm{Na} \%}{\mathrm{Na}+\mathrm{Ca}+\mathrm{Mg}+\mathrm{K}}$

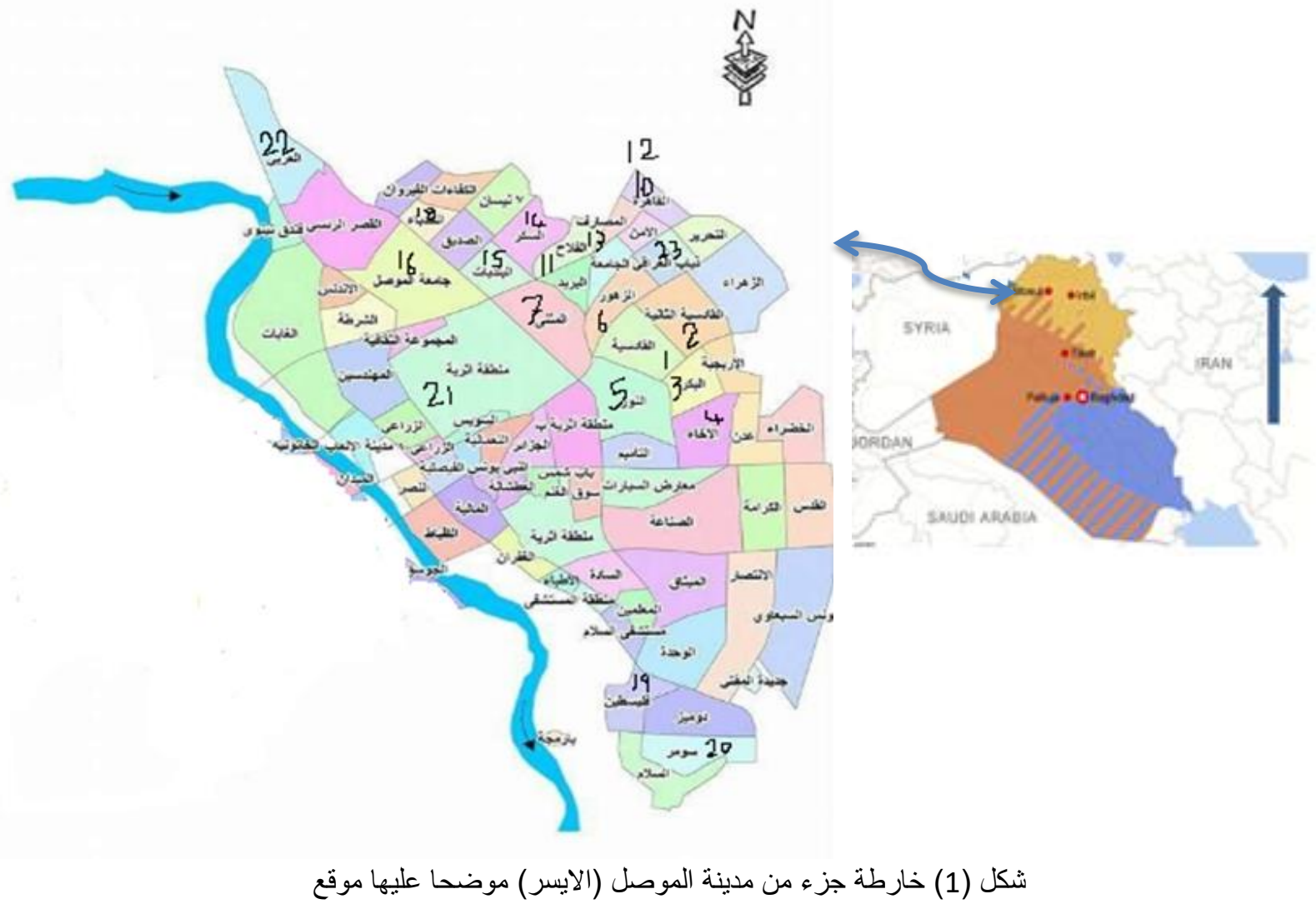


Results and discussion : النتائج والمناقشة ان جيولوجية منطقة الدراسة تقع ضمن ترسبات تكوين الفتحة , والذي تتميز بطبقة بلاسبي Plaspi , تتنشر فيها الصخور القابلة للذوبان مثل صخور الجبسوم والانهايدرايت والهاليت والمارل مما تؤثر في نوعية المياه وتزيد من تركيز الاملاح الذائبة فيها[7]]. و [18]. درجة حرارة الماء: تتاثر درجة حرارة المياه السطحية بتغير درجة حرارة الهواء , على عكس المياه الجوفية البعيدة تقريبا عن التغاير الذي يحصل في درجة حرارة الهواء لذا يكون مدى التغاير قليلا [14].

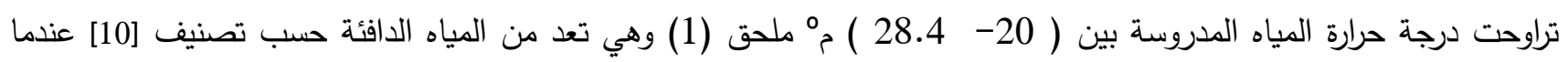

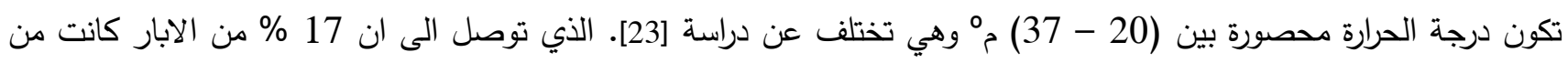

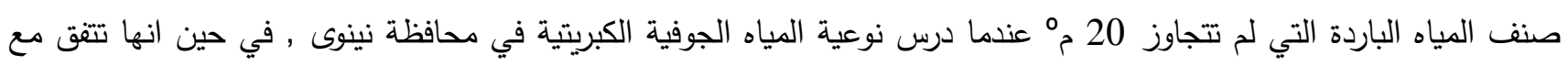

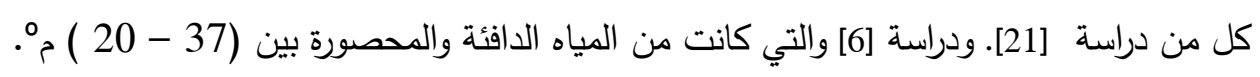

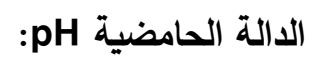
تميل معظم المياه المدروسة نحو القاعدية وتراوحت قيمها بين ( 7.02 - 78. 7 ) ملحق (1) , وبصورة عامة ان اغلب المياه

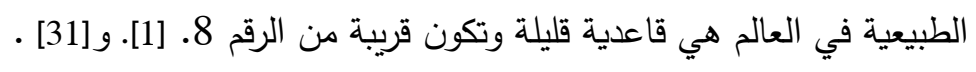
بينت النتائج ان 22\% من مياه الابار المدروسة هي من نوع معتدلة و 78\% هي ضعيفة ونئة القاعدية , حيث تكون معتدلة عندما

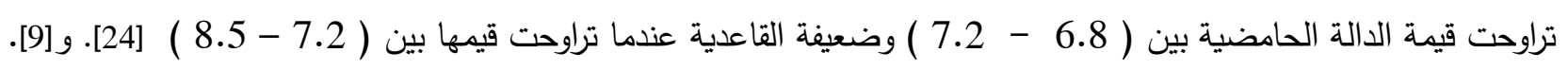

\section{التوصيلة الكهربائية Electrical Conductivity: تراودئه}

يبين ملحق ( 1 ) ان اعلى قيمة للتوصيل الكهربائي ( 2456 ) مايكروموز / سم عند البئر 19, في حين اقل قيمة سجلت (791)

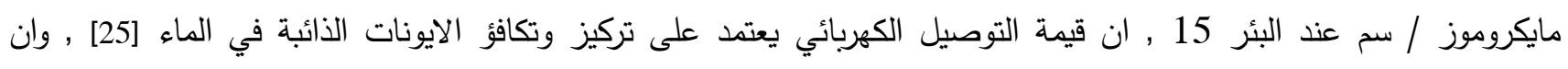

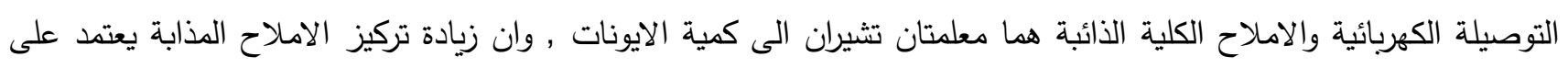
التركيب الجيولوجي لمنطقة الدراسة [3].

الاملاح الكلية الذائبة Total Dissolved Salts:

يلعب التركيب الجيولوجي لمنطقة الدراسة دورا مهما في زيادة تركيز الاملاح الذائبة حيث تتكون من صخور الانهايدرايت والجبسوم مما يؤدي الى زيادة ملوحة مياه الابار المدروسة روتتكون هذه الاملاح الذائبة الكلية من مجموع الايونات السالبة والموجبة [8].

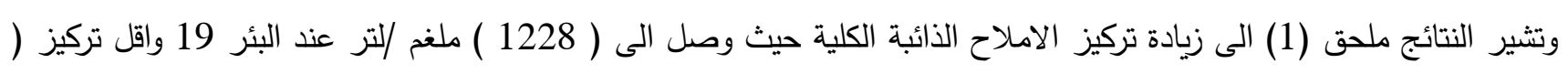
406 ) ملغم / لتر عند البئر 15 .

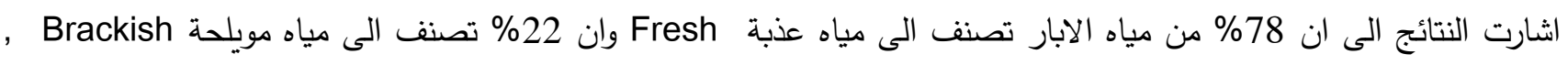

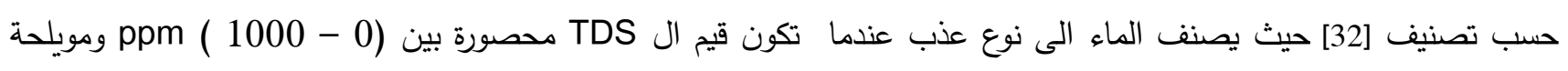

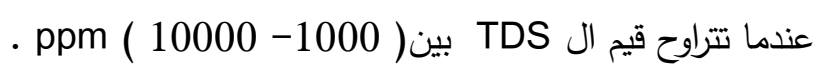

الاوكسجين المذاب Disolved Oxygen: يعتمد تركيز الاوكسجين المذاب على عدة عوامل منها انه كلما زاد تركيز الاملاح المذابة قلة تركيزه [30] , وهذا ما نلاحظه في

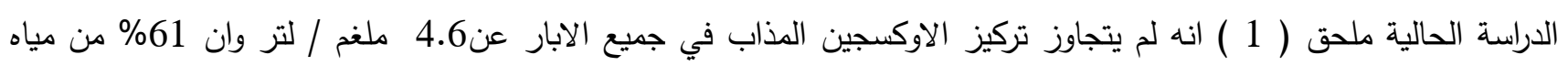

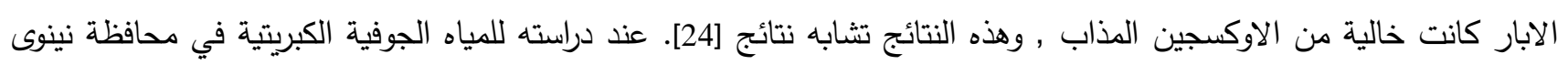

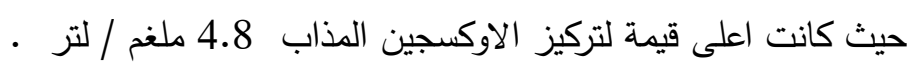


ايوني الكاليسيوم والمغنيسيوم Calcium and Magnesium ions: ان من مسببات الرئيسية لعسرة الماء في الطبيعة هو ايوني الكالسيوم والمغنيسيوم , ان تركيز ايون الكالسيوم هو اكثر من تركيز

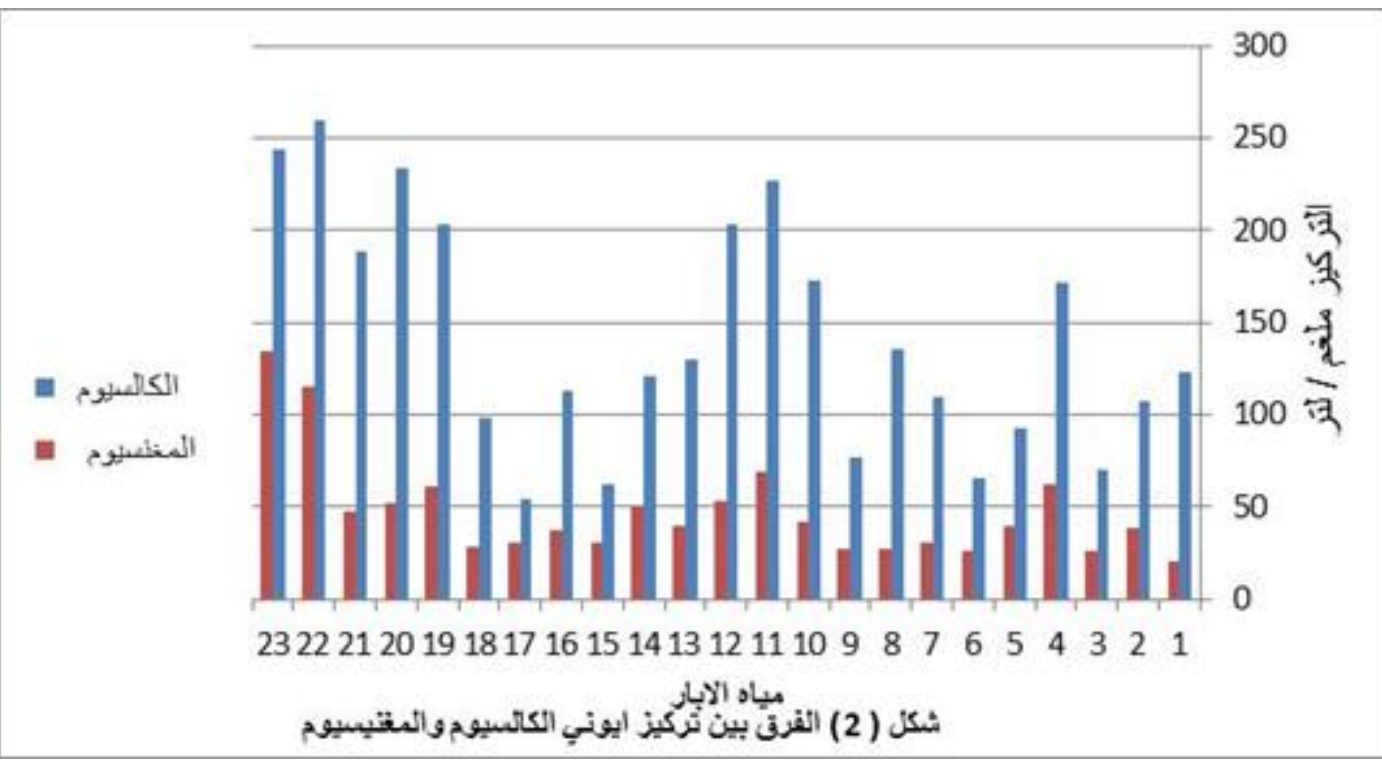

المغنيسيوم في جميع الانظمة المائية , قد يعود هذا الاختلاف في التركيز الى اختلاف التركيب الجيولوجي للمناطق او قد يعود الى الى ميل الكالسيوم الى التقاعل مع ثنائي اوكسيد الكاربون الذائب اكثر من ميله للتفاعل مع ايون المغنيسيوم [25], وهذا التفاوت في التركيز للايونين ينطبق في البحث الحالي حيث تركيز ايون الكالسيوم اكبر من تركيز ايون المغنيسيوم في جميع مياه الابار قيد

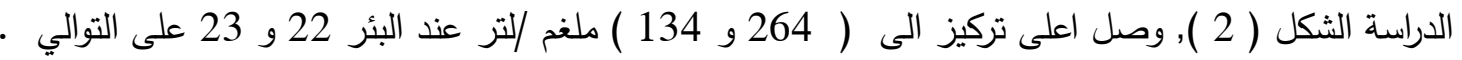

ايوني الصوديوم والبوتاسيوم Sodium and Potassium ions: يبين ملحق ( 1 ) ان تركيز ايوني الصوديوم والبوتاسيوم تراوح بين ( 16 - 89 ) ملغم / لتر و (0.1 - 13.5 ) ملغم / لتر • على التوالي ملى يوجد تقارب كبير بين الايونين البوتاسيوم والكالسيوم في سلوكهما الايوني الا ان تركيز البوتاسيوم في الطبيعة اقل من تركيز الصوديوم واكدة الدراسة الحالية ذلك في شكل (3) , ان وفرة المتبخرات في التركيب الجيولوجي لمنطقة الدراسة يزيد من تركيز الصوديوم وتتميز بذوبانها الثديد في المياه مقارنة بايون البوتاسيوم بالرغم من وفرة الاخير في القشرة الارضية [19].

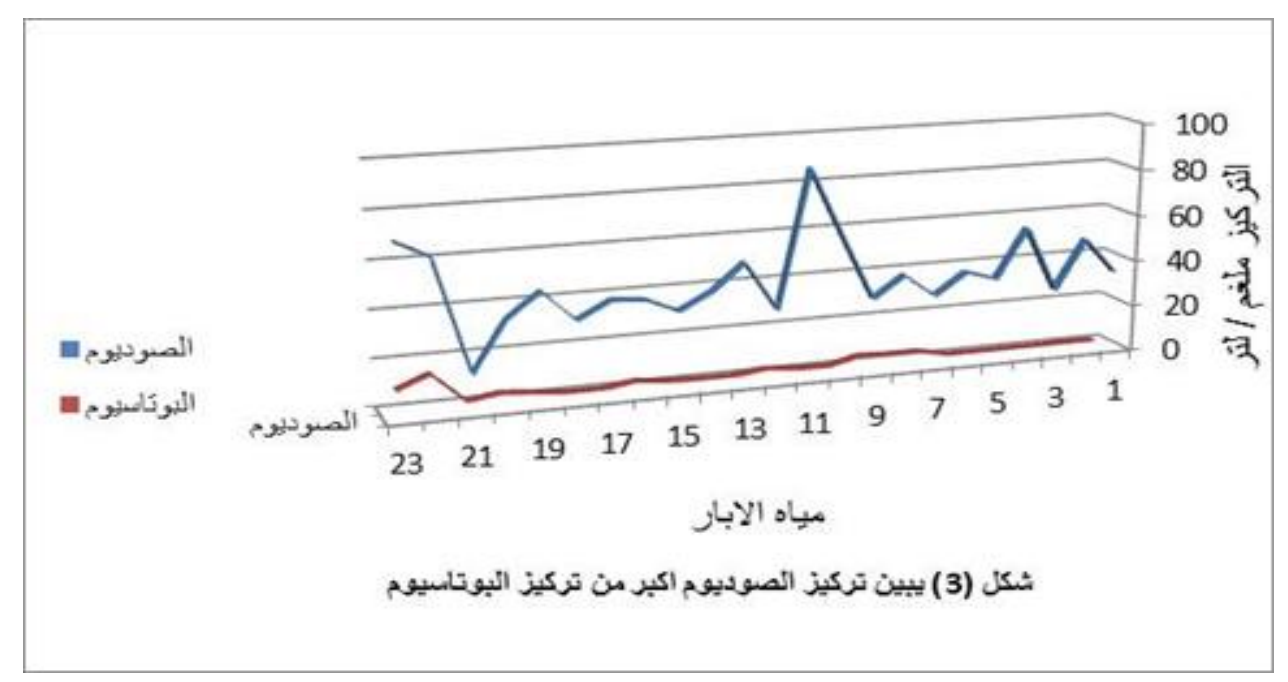


ايون الكبريتات Sulfate ion: ان مصدر الكبريتات في المياه الجوفية ناتج من ذوبان الصخور المكونة لتكوين انجانة مثل صخور الجبسوم والانهايدرايت , فضلا عن اكسدة خامات الكبريتيد [4]. يبين ملحق ( 1 ) ان تركيز الكبريتات في المياه المدروسة تراوح بين (25 - 13037 ) ملغم / لتر في البئرين 1 و 14 على • التوالي

: Nitrate ion ايون النترات ان زيادة تركيز النترات في المياه السطحية او الجوفية قد يعود الى اكسدة المواد العضوية الناتجة من تحلل وتفسخ المركبات

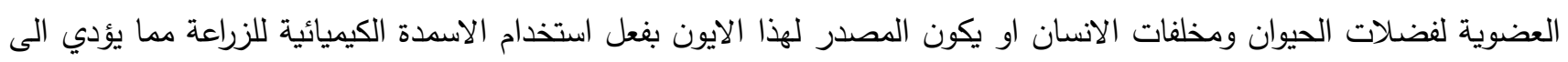
انجرافها الى المياه , وان سبب انخفاض تركيز النترات في المياه الجوفية قد يعود الى عدم وصول مسببات التلوث اعلاه الى المياه الجوفية مقارنة مع المياه السطحية [19]. يشير الملحق ( 1 ) الى انخفاض تركيز النترات في المياه الجوفية حيث سجل ادنى تركيز ( 0.1 ) ملغم / لتر في البئر 4 في

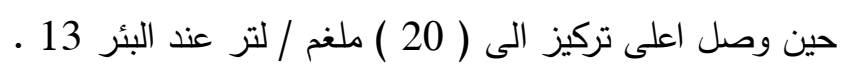

ايون الكلورايد Chloride ion:

ان تركيز ايون الكلورايد مرتفعة حيث وصل اعلى تركيز الى ( 204 ) ملغم / لتر واقل تركيز (32 ) ملغم / لتر عند البئرين 11 مالت و 7 على التوالي , ان التركيز العالي لهذا الايون قد يعود الىى وفرة صخور المتبخرات لمنطقة الدراسة بسبب معظم املاحه سريعة الذوبان [21] و [23]

\section{ايون الفوسفات Phosphate ion}

ان عملية حجز الفوسفات الذائبة بامتزازها من قبل حبيبات التربة او ترسيبها بشكل فوسفات الكالسيوم او الاثثين معا تصبح النتيجة

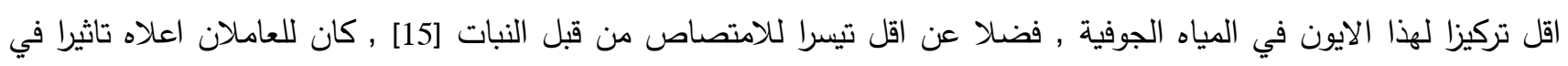
انخفاض تركيز الفوسفات الذائبة في المياه المدروسة حيث تراوح تركيز الفوسفات في مياه الابار بين ( 0.015 - 0.072 م 0.0 ) ملغم

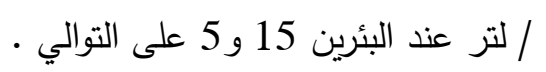

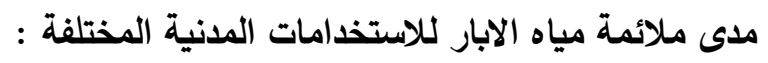

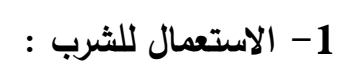

يبين ملحق (1) ان قيمة التوصيل الكهربائي لمياه الابار قيد الدراسة تراوحت بين (791 -2465 ) مايكروموز / سم ,

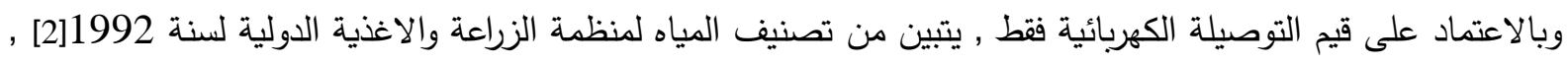

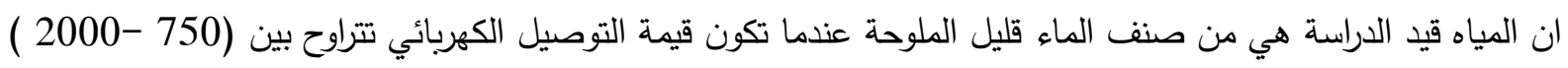

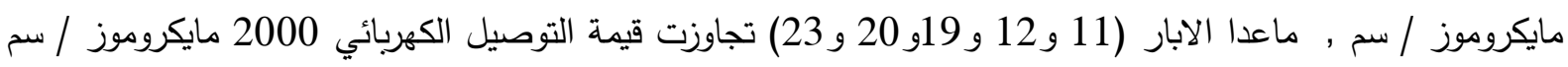
وهي من صنف مياه متوسط الملوحة و جميع الابار هي غير صالح للشرب لتجاوز قيم التوصيل الكهربائي لمياه الابار

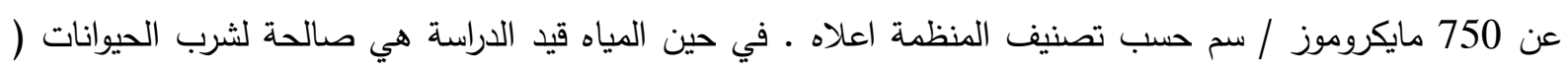

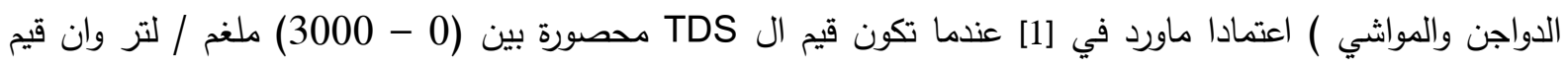

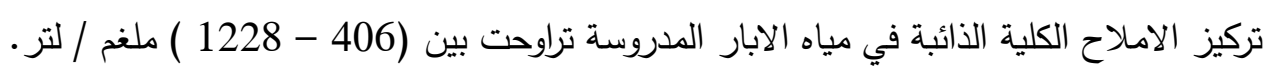


2- 2 الاستخدام للري :

تتاثر الزراعة بنوعية مياه الري ,حيث تؤدي التراكيز العالية للاملاح في الماء الى تراكمها في التربة وبالتالي تؤثر في نمو النبات [5]. تتواجد نظم تصنيفية لمياه الري حسب ماورد [1] و [27] وهي:-

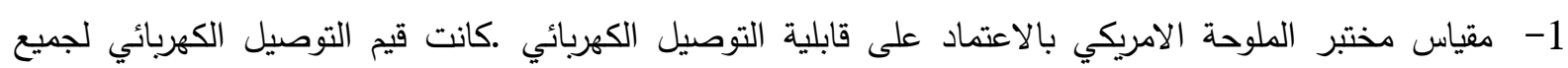

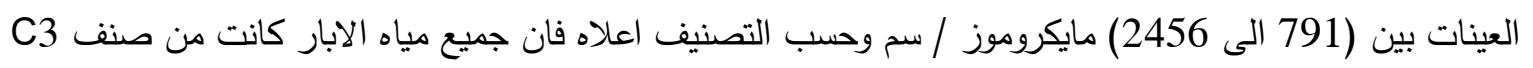

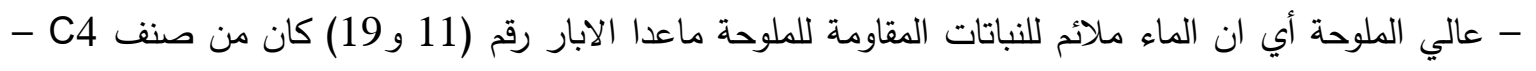

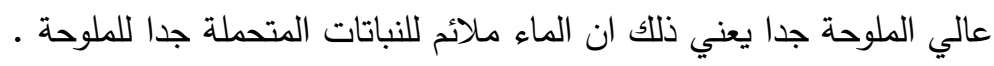

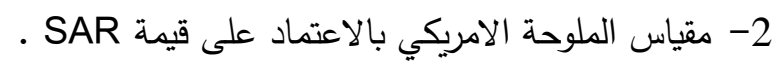

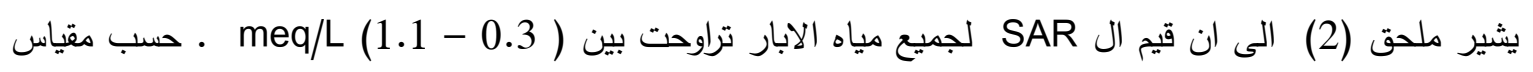

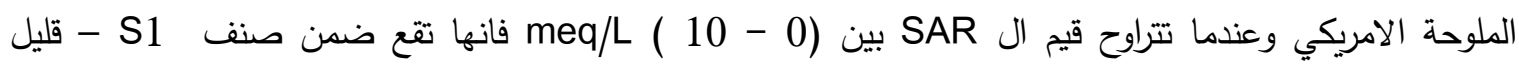

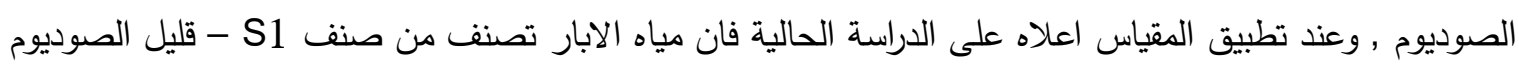

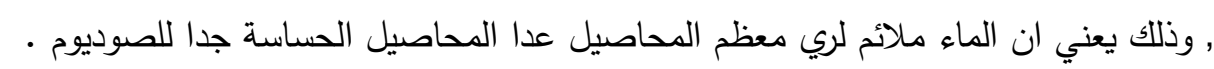

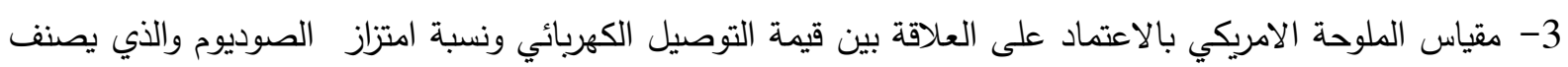
الماء الى 16 رتبة . عند تطبيق العلاقة اعلاه لعينات الدراسة الحالية فان جميع مياه الابار تقع ضمن الرتبة ( C3 - S1 ) بمعنى

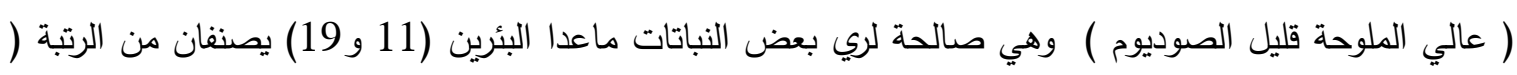

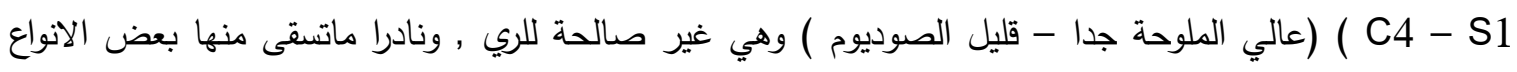
النباتية . 4- تعتمد محددات نوعية مياه الري على عدة عوامل منها الصودية, ومن مؤشرات الصودية للتعبير عن خطورته في الري

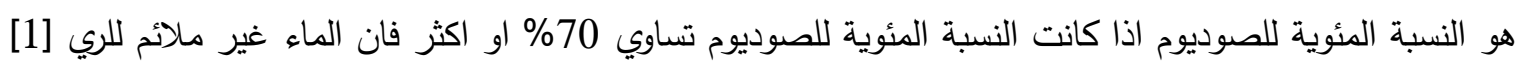

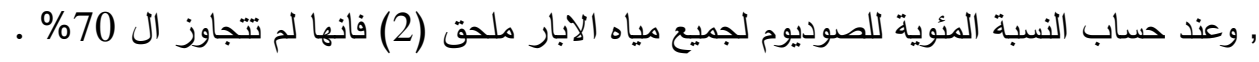


Journal of Education and Science (ISSN 1812-125X), Vol: 30, No: 5, 2021 (54-64)

\begin{tabular}{|c|c|c|c|c|c|c|c|c|c|c|c|c|c|c|}
\hline \multicolumn{15}{|c|}{ ملحق (1) الصفات الفيزياوية والكيمياوية لمياه الابار } \\
\hline ملغم / لتر & ملغم / لتر & ملغم / لتر & ملغم / لتر & ملغم / لتر & لمغوديوم & ملغم / لمغنسيوم & ملغم / لترالسيوم & الاوكسجين & الألائبة & $\begin{array}{c}\text { E.C } \\
\text { مايكروموز سم }\end{array}$ & $\mathrm{pH}$ & درجة & الحي السكني & البئر \\
\hline 0.051 & 9 & 25 & 88 & 0.1 & 35 & 20 & 123 & 0.0 & 665 & 1327 & 7.35 & 27 & القادسية الاولى القى & 1 \\
\hline 0.025 & 9 & 486 & 118 & 0.3 & 50 & 39 & 107 & 1 & 823 & 1655 & 7.11 & 27 & القادسية الثانية & 2 \\
\hline 0.025 & 6 & 633 & 50 & 0.1 & 29 & 26 & 70 & 0.0 & 447 & 895 & 7.15 & 25 & البكر & 3 \\
\hline 0.0186 & 0.1 & 211 & 90 & 0.1 & 57 & 62 & 172 & 0.0 & 966 & 1941 & 7.75 & 27 & الاخاء & 4 \\
\hline 0.072 & 0.7 & 313 & 44 & 0.1 & 40 & 40 & 93 & 1.2 & 970 & 1941 & 7.67 & 26 & النور & 5 \\
\hline 0.027 & 7 & 74 & 54 & 0.1 & 40 & 26 & 66 & 0.8 & 484 & 958 & 7.66 & 25 & الزهور & 6 \\
\hline 0.035 & 2 & 485 & 32 & 2.9 & 31 & 30 & 109 & 0.9 & 578 & 1156 & 7.47 & 23 & المثنى & 7 \\
\hline 0.020 & 5 & 412 & 96 & 2.9 & 41 & 27 & 136 & 0.0 & 714 & 1428 & 7.30 & 21.4 & المشر اق & 8 \\
\hline 0.060 & 9 & 403 & 62 & 3.5 & 32 & 27 & 77 & 0.0 & 493 & 991 & 7.23 & 26 & البريد & 9 \\
\hline 0.022 & 13 & 897 & 160 & 0.3 & 60 & 42 & 173 & 0.0 & 975 & 1971 & 7.22 & 25 & القاهرة & 10 \\
\hline 0.019 & 18 & 1613 & 204 & 0.6 & 89 & 69 & 227 & 0.0 & 1142 & 2294 & 7.38 & 25 & فلاح الثانية & 11 \\
\hline 0.016 & 18 & 1136 & 158 & 1.9 & 31 & 53 & 203 & 0.0 & 1060 & 2115 & 7.36 & 24 & العباسية & 12 \\
\hline 0.019 & 20 & 807 & 90 & 0.1 & 52 & 40 & 130 & 0.0 & 756 & 1494 & 7.45 & 24 & فلاح الاولى & 13 \\
\hline 0.019 & 19 & 3037 & 66 & 0.1 & 41 & 50 & 121 & 0.0 & 736 & 1478 & 7.30 & 26 & السكر & 14 \\
\hline 0.015 & 6 & 346 & 26 & 0.8 & 34 & 31 & 62 & 0.0 & 406 & 791 & 7.78 & 24 & البلديات & 15 \\
\hline 0.022 & 10 & 461 & 60 & 2.2 & 40 & 37 & 113 & 0.0 & 686 & 1372 & 7.39 & 25 & كفاءات الاولى & 16 \\
\hline 0.018 & 11 & 230 & 50 & 0.1 & 41 & 31 & 54 & 1.3 & 491 & 1036 & 7.50 & 27 & شقق الخضر اء & 17 \\
\hline 0.016 & 10 & 387 & 52 & 0.1 & 34 & 28 & 98 & 0.0 & 508 & 1006 & 7.30 & 23 & الحدباء & 18 \\
\hline 0.017 & 0.8 & 101 & 48 & 1.5 & 47 & 61 & 203 & 1 & 1228 & 2456 & 7.13 & 24 & فلسطين & 19 \\
\hline 0.017 & 0.5 & 86 & 34 & 2.8 & 37 & 52 & 234 & 1.5 & 1057 & 2112 & 7.41 & 28 & سومر & 20 \\
\hline 0.018 & 2 & 101 & 46 & 0.8 & 16 & 48 & 189 & 2.3 & 908 & 1816 & 7.02 & 25 & نركال & 21 \\
\hline 0.016 & 3 & 37 & 84 & 13.5 & 64 & 115 & 260 & 1.2 & 749 & 1499 & 7.13 & 23 & العربي & 22 \\
\hline 0.020 & 9 & 89 & 174 & 8.1 & 71 & 134 & 244 & 0.0 & 1071 & 2160 & 7.25 & 24 & الرشيدية & 23 \\
\hline
\end{tabular}




\begin{tabular}{|c|c|c|c|c|c|c|c|}
\hline \multicolumn{8}{|c|}{ ملدق (2) تركيز الايونات الموجبة ( ملي مكافئ / لتر , meq ) } \\
\hline $\mathrm{Na} \%$ & SAR & المجموع & البوتاسيوم & الصوديوم & المغنسيوم & الكالسيوم & رقم البئر \\
\hline 16 & 0.75 & 9.2 & 0.0025 & 1.50 & 1.6 & 6.1 & 1 \\
\hline 20 & 1.0 & 10.7 & 0.0076 & 2.18 & 3.2 & 5.3 & 2 \\
\hline 19 & 0.7 & 6.7 & 0.0025 & 1.25 & 2.1 & 3.5 & 3 \\
\hline 15 & 0.95 & 16.1 & 0.0025 & 2.47 & 5.1 & 8.6 & 4 \\
\hline 16 & 0.8 & 9.5 & 0.0025 & 1.56 & 3.3 & 4.6 & 5 \\
\hline 21 & 0.9 & 6.9 & 0.0025 & 1.47 & 2.1 & 3.3 & 6 \\
\hline 14 & 0.7 & 9.3 & 0.074 & 1.33 & 2.5 & 5.4 & 7 \\
\hline 16 & 0.9 & 10.9 & 0.074 & 1.78 & 2.2 & 6.8 & 8 \\
\hline 19 & 0.8 & 7.1 & 0.089 & 1.37 & 2.2 & 3.4 & 9 \\
\hline 18 & 1 & 14.7 & 0.007 & 2.61 & 3.5 & 8.6 & 10 \\
\hline 19 & 1.3 & 20.9 & 0.015 & 3.88 & 5.7 & 11.3 & 11 \\
\hline 8 & 0.5 & 15.9 & 0.048 & 1.33 & 4.4 & 10.1 & 12 \\
\hline 19 & 1 & 12.1 & 0.002 & 2.25 & 3.3 & 6.5 & 13 \\
\hline 15 & 0.8 & 12 & 0.002 & 1.80 & 4.1 & 6.0 & 14 \\
\hline 20 & 0.9 & 7.2 & 0.020 & 1.47 & 2.6 & 3.1 & 15 \\
\hline 25 & 1.1 & 7 & 0.002 & 1.78 & 2.5 & 2.7 & 16 \\
\hline 17 & 0.8 & 8.7 & 0.002 & 1.49 & 2.3 & 4.9 & 17 \\
\hline 12 & 0.8 & 17.2 & 0.038 & 2.03 & 5.0 & 10.1 & 18 \\
\hline 9 & 0.6 & 17.7 & 0.071 & 1.62 & 4.3 & 11.7 & 19 \\
\hline 5 & 0.3 & 14.0 & 0.020 & 0.71 & 3.9 & 9.4 & 20 \\
\hline 11 & 0.8 & 25.6 & 0.345 & 2.76 & 9.5 & 13 & 21 \\
\hline 12 & 0.9 & 26.5 & 0.207 & 3.07 & 11.0 & 12.2 & 22 \\
\hline 17 & 0.8 & 10.4 & 0.056 & 1.75 & 3.0 & 5.6 & 23 \\
\hline
\end{tabular}

الاستتاجات : conclution

1- تركيز الاوكسجين المذاب لم تتجاوز في جميع العينات عن 4.6 ملغ / لتر .

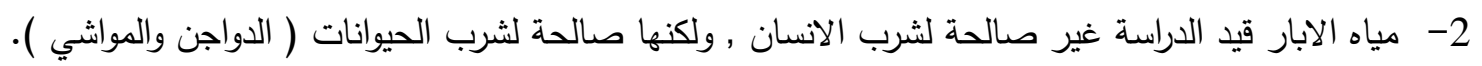

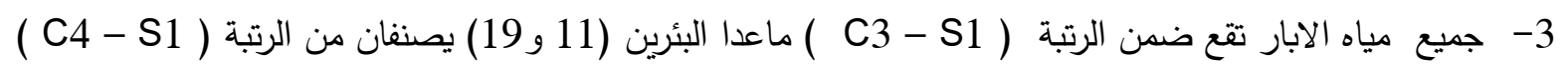

شكر وتقدير Acknowledgement:

الثكر والتقدير الى عمادة كلية العلوم ورئاسة قسم علوم الحياة والتدريسين في القسم على دعمهم لي وما قدموا من تسهيلات لإنجاز

\section{المصادر:references:}

1. Abbawi, Suad Abd and Hassan. Muhammad Salman., "Practical Engineering of the Environment and Water Testing," Dar Al-Hikma Printing and Publishing, University of Mosul (1990). ( in Arabic)

2. Abdel Hamza, Jabbar Sellal., Al-Qadisiyah Journal of Agricultural Sciences vol.5, no.2,pp.62-72, (2015) ( in Arabic).

3. Abdulhussein, Firas Mudhafar., Iraqi Journal of Science. vol.59, no.1,pp.135-143, (2018). ( in Arabic) 
4. Abdullah, Fakhri Khalaf and Hussain., Dhamia Adham."A study of the physical and chemical properties of well water in the district of Samarra," Journal of the College of Education for Girls, vol. 26, no.2, pp. 402-417, (2015)

5. Abu Glida, Intisar Emahmed and Samhoud, Fawzia Al-Mabrouk., Science Magazine in Misurata Libya. A special issue of the second annual conference on the theories and applications of basic and biological sciences. University of. pp. 184-191, (2018) (in Arabic)

6. Al-Badrani, Hawazen Hassan Fathi Majoul., M.Sc.Thesis,College of Environment. Mosul University (2017). ( in Arabic)

7. Al-Barzanji, Ahmad Jaafar Ahmad., M.Sc.Thesis, College of Education for Pure Sciences, University of Mosul (2020).

8. Al-Hassan, Shukri Ibrahim and Al-Badri, Ali Zaeef Tayeh., Dhi Qar University Journal, vol. 14, no.2, pp. 157-180, (2019). ( in Arabic)

9. Ali, Amer Abdul Rahim Muhammad.,M.Sc.Thesis, College of Science, University of Mosul, (1989). ( in Arabic)

10. Al-Jubouri, Mahmoud Ismail and Al-Hamdani, Ibrahim Omar Saeed and Erhail, Hamid Mahdres., Tikrit Journal of Pure Sciences, vol.17, no.1, pp. 25-31, (2012). ( in Arabic).

11. Al-Khashab, and Wafiq Hussein., "Geomorphology: definition, development, and applied fields," First part. University of Baghdad, (1978). ( in Arabic).

12. Al-Obeidi, Basem Hussain Khudair and Salman Muhammad Sadiq.,Al-Nahrain University Journal, vol.14, no.1, pp. 8-16, (2011). ( in Arabic).

13. APHA,."Standard Methods for the Examination of Water and Waste Water Analysis,".14 ${ }^{\text {th }}$ Ed. American Public Health Association. New York, (1976).

14.

Assaf, Hoda and Al-

Masry, Muhammad Saeed.,"Groundwater Pollution Sources,". Syrian Atomic Energy Comm Damascus (2007). ( in Arabic).

15 .

Atwi, Ali Ahmed,

Technical Journal, Technical Education Authority, vol.24, no. 1, pp.137-146, (2011). ( in Arabic). 16.

Buran, Alia Hatog and

Abu Dayya, Muhammad Hamdan,. "Ecology," Dar Al Shorouk Press - Amman, (1993). ( in Arabic).

17.

Dellys, Israa Salman, Tikrit University Journal of Agricultural Sciences, vol.17, no.2, pp.188-201, (2017). ( in Arabic). 18.

Dhyaaldain K,. Iraqi Journal of Science, vol.56, no. 2C, pp.1763-1769, (2015).

19. Hussein, Ruaa Ali and Al-Salem, Taha Hussein Ali,.. Kirkuk University Journal /Scientific Studies, vol.12, no.1,pp. 268-290, (2017).

20. Kanna, Abdel Moneim Muhammad Ali and Saeed, Salah Muhammad,. Al-Qadisiyah Journal of Pure Sciences, vol.19, no.2, pp.40-54, (2014). ( in Arabic).

21. Kanna, Abdel Moneim Muhammad Ali and Yaqoub, Hala Muzhar and Muhammad, Abdullah Khudair,. Tikrit Journal of Pure Sciences, vol. 22, no.10, pp. 22-29, (2017). ( in Arabic).

22. Kanna, Abdel Moneim Muhammad Ali,. Tikrit Journal of Pure Sciences. vol. 2, no.11, pp.134-138, (2006) (in Arabic).

23. Kanna, Abdel-Moneim Muhammad Ali,. Rafidian Sciences Journal, vol.27, no.5, pp. 212222, (2018). ( in Arabic).

24. Kanna, Abdel-Moneim Muhammad Ali., M.Sc.Thesis, College of Science, University of Mosul, (2001). ( in Arabic). 25.

Khether, Lec. Sura I.

Khalaf, Lec. Hanaa H. and Hassan, Ikhlas T., Journal of Basic Education College, vol.15, no.3, pp.1989-2002,(2019). 
26. Muhammad, Samira Faydallah Kirkuk University Journal of Agricultural Sciences, vol.9, no.4, pp.126-136, (2018).

27. Richards,L.A,."Diagnosis and improvement of saline and alkali soils". Handbook 60 .Washington,D.C, (1954).

28. Saeed, Ibrahim Omar Al-Rafidain Science Journal, vol.25, no.1, pp.79-96 (2014). ( in Arabic).

29. Shehab, Abd al-Muhsin Sa'dallah and al-Abd Rabu, Walid Muhammad Shit and Khumayl Ahmad,. Tikrit Journal of Engineering Sciences, vol.20, no.3, pp. 33-16 (2017). ( in Arabic).

30. Shekha, Yahya Ahmed . Ali, Luay Abdul-Qader and Toma, Janan Jabbar, vol. 14, no.2, pp. 335-342, (2017).

31. Talling , J.F,. "Water characteristics in Euphrates \&Tigris Mesopotamian," 1 E.D. vol.38,(1980).

32. Todd, D.K.,"Ground water Hydrology," $2^{\text {nd }}$. Wiley, New York, (1980). 